\title{
Why some patients are keeping their heads down
}

\section{Rosamund Snow and David Gilbert detect a sea change in the way patients and the public have started talking about the junior doctors'strike}

\section{Rosamund Snow patient editor, David Gilbert patient panel member}

The BMJ

We first noticed the difference early on 26 April. On Twitter, groups of patients were asking each other what they thought about the strike, rather than stating a definite opinion. People discussed whether it was time to stop self censoring.

Increasingly, they seemed to be turning to direct messaging or private emails to have these discussions.

The overwhelming feeling was that no patients in these groups wanted to go on record with their views. We wanted to get patients' voices into The BMJ for its coverage of the junior doctors' strike, and a request for views resulted in immediate volunteers among the enthusiastic pro-strike patients-but every conversation we had about featuring more diverse responses to the strike ended the same way. Long, detailed, passionate emails full of interesting thoughts, closing with something like, “. . . but I don't want to put this into print."

In short, anyone using mainstream or even social media to gauge public opinion on the strike is going to miss a lot. Don't be fooled: a murmur is going on below the parapet. Some of the main themes coming up in these conversations are:

- We are not comfortable with doctors and politicians discussing our health service without patients being involved.

- We are not happy that "patient safety" and "patient harm" are being defined by doctors and politicians but not by those at risk of harm.
- We are afraid that questioning anything in the debate will lead to a backlash.

- We no longer know where to find trustworthy information on this topic.

- We are worried that our experiences will be taken out of context and used by one side to bash the other; we are concerned that the debate is now so polarised that any nuance will get shouted down; we are afraid of becoming political pawns.

- We want to help, but we don't know how. Calls for patients to mediate in this dispute or discuss what we mean by "safety" or "24/7 working" have been made very eloquently, for example by National Voices, and patients are talking about how we could resolve this. Nobody is taking us up on it.

What is clear is that these closed discussions are not saying, "My doctor, right or wrong," but nor are they pro-government. One comment (reproduced by permission) on a closed Facebook page sums it up: "I support the junior doctors. I don't support this strike. I really don't support Hunt. How am I going to fit all that on a banner?" 